\title{
BMJ Open Predictors of pain reduction following manual therapy in patients with temporomandibular disorders: a protocol for a prospective observational study
}

\author{
Giacomo Asquini, ${ }^{1,2}$ Andrea Edoardo Bianchi, ${ }^{2}$ Nicola R Heneghan (D) , \\ Alison B Rushton (D) , ${ }^{1}$ Giulia Borromeo, ${ }^{2}$ Matteo Locatelli, ${ }^{3}$ Deborah Falla (D) ${ }^{1}$
}

To cite: Asquini G, Bianchi AE, Heneghan NR, et al. Predictors of pain reduction following manual therapy in patients with temporomandibular disorders: a protocol for a prospective observational study. BMJ Open 2019;9:e032113. doi:10.1136/ bmjopen-2019-032113

- Prepublication history and additional material for this paper are available online. To view these files, please visit the journal online (http://dx.doi. org/10.1136/bmjopen-2019032113).

Received 03 June 2019 Revised 11 October 2019 Accepted 15 0ctober 2019

Check for updates

(c) Author(s) (or their employer(s)) 2019. Re-use permitted under CC BY-NC. No commercial re-use. See rights and permissions. Published by BMJ.

${ }^{1}$ Centre of Precision

Rehabilitation for Spinal Pain (CPR Spine), School of Sport, Exercise and Rehabilitation Sciences, University of Birmingham, Birmingham, UK ${ }^{2}$ Department of

Craniomandibular Disorders, Italian Stomatologic Institute,

Milan, Italy

${ }^{3}$ Department of Rehabilitation and Functional Recovery, IRCCS San Raffaele Scientific Institute, Milan, Italy

Correspondence to Professor Deborah Falla; d.falla@bham.ac.uk

\section{ABSTRACT}

Introduction Temporomandibular disorders (TMDs) are principally characterised by pain in the craniomandibular area and probable limitations of jaw opening. Manual therapy, like other recommended conservative treatments included in clinical guidelines, is commonly used to treat patients with TMD to reduce pain and improve function. However, outcomes may be variable. The aim of this study is to identify predictors associated with pain reduction in patients with TMD following manual therapy by analysing a combination of patient-reported outcome measures and clinical tests. Such knowledge will support a more personalised management approach by facilitating clinical decision-making.

Methods/analysis An observational prospective design will recruit a cohort of 100 adults with a diagnosis of TMD (according to Axis I of the Diagnostic Criteria for TMD) at a Dental Hospital in Italy. Patients will be treated with four weekly sessions of manual therapy applied to craniomandibular structures. An array of predictors has been chosen based on previous research on prognostic factors for TMD and altered pain modulation in musculoskeletal disorders. Candidate predictors including demographic variables, general health variables, psychosocial features, TMD characteristics and clinical tests of the temporomandibular joint and masticatory muscles will be collected at baseline. Definition of good outcome is a clinically significant reduction of pain intensity over the last week ( $\geq 30 \%$ reduction Visual Analogue Scale) immediately following the four week intervention. Exploratory factor analysis will be applied to analyse factor loading of candidate predictors for good outcome at four weeks. Subsequently, a logistic multivariable regression model will be performed to calculate low and high risk of good outcome.

Ethics and dissemination Ethical approval has been obtained from the 'Fondazione IRCCS Ca' Granda Ospedale Maggiore Policlinico' and University of Birmingham Ethics Committee. The results will be submitted for publication in a peer-reviewed journal and presented at conferences. Trial registration number NCT03990662; Pre-results.

\section{INTRODUCTION}

Temporomandibular disorders (TMD) affect $\sim 10 \%$ of the adult population and, in the
Strengths and limitations of this study

- This will be the first study to identify predictors associated with pain reduction following manual therapy interventions in patients with temporomandibular disorder (TMD).

- The study will use a comprehensive array of candidate factors to predict clinically relevant pain reduction.

- The implications from this study will facilitate clinical decision-making for manual therapists managing patients with TMD.

- Alternative or additional predictors could be valuable to include but the candidate predictors have been prioritised as they are reliable and valid measures which have a relationship with pain.

- The study could potentially generate a nonrepresentative sample of patients as it will exclude people who have already received recent treatment for their TMD.

USA alone, are estimated to cost US $\$ 4$ billion per year on management. ${ }^{1}$ In Spain, the incidence of TMD has significantly increased (from $8 \%$ in 1993 to $14 \%$ in 2015) despite a clear improvement in general oral health over the entire period. ${ }^{3}$ Although some countries report less prevalence of TMD such as in Sweden $(\sim 5 \%),{ }^{4}$ TMD remains a public health-related challenge. TMD are principally characterised by pain and limitations of jaw opening ${ }^{5}$ but many patients also complain of neck and back pain or pain at other sites. ${ }^{6}$

Physical therapy is one of the most common conservative interventions for the management of $\mathrm{TMD}^{7}$ and given that the aetiology may be unclear, ${ }^{8}$ several therapeutic approaches have been described. ${ }^{9}$ One approach is manual therapy applied to the craniomandibular structures with evidence suggesting a significant reduction in pain with manual therapy treatment, ${ }^{10}$ although responses are highly variable. ${ }^{11}$ In 
other musculoskeletal pain disorders, such as neck or back pain, pain reduction from manual therapy has been shown to be superior to other treatments (eg, therapeutic exercise) when targeted towards patients with specific clinical features including the onset of symptoms within 30 days. ${ }^{12} 13$ Nevertheless, in TMD, no previous study has investigated patient factors associated with significant pain reduction following manual therapy. Such knowledge could be achieved by identifying potential predictors (eg, pain characteristics, psychosocial features, TMD characteristics) of pain reduction following manual therapy interventions in patients with TMD to support a more personalised management approach.

Very few studies have examined factors associated with pain reduction in patients with TMD. Forssell et al conducted a prospective cohort study with 263 primary care patients with TMD pain. ${ }^{14}$ They analysed several potential predictors of persistent pain at 1-year follow-up including demographic, pain-related and psychosocial variables. It was concluded that patients with TMD who have had numerous previous healthcare visits, complained of high-intensity pain at other body sites and had a greater number of disability days, were at greater risk of having pain 1 year after the initial assessment. Nevertheless, this study did not examine predictors of pain reduction related to a therapeutic intervention which could be useful to inform clinical practice. Kapos et al investigated the association of long-term pain intensity with baseline health-related quality of life and jaw functional limitation in patients with TMD. ${ }^{15}$ Findings suggested that baseline health-related quality of life is inversely proportional with pain intensity at an 8-year follow-up regardless of the type of treatment that they received (eg, surgery, drugs, physical therapy or unconventional therapy). After adjusting for the type of treatments received, by clustering the participants into three groups (medical/conventional management, complementary medicine and surgical intervention), each predictor analysed (demographic, pain-related and health-related quality of life) maintained similar statistical significance. Notwithstanding, the group classified as 'medical/conventional management' included participants receiving diverse treatments ranging from physical therapy, pharmacology (acetaminophen, antidepressants, anti-inflammatories) to the application of a mouth appliance (eg, Michigan splint). This previous work can facilitate clinicians to identify patients who are more challenging to treat by identifying clinical features associated with persistent pain in the long-term regardless of the type of interventions applied. However, currently no study has examined predictive factors associated with pain reduction following manual therapy interventions in patients with TMD.

The aim of this study is to identify predictors associated with pain reduction in patients with TMD following manual therapy applied to craniomandibular structures by analysing a combination of: (1) demographical variables, (2) general health variables, (3) psychosocial features, (4) TMD characteristics and (5) clinical tests of the temporomandibular joint and masticatory muscles. The knowledge gained from this study will facilitate clinical decision-making for manual therapists managing patients with TMD by providing clinicians with key factors to evaluate, to determine whether or not the patient is likely to have a clinically relevant reduction in their pain immediately following 4 weekly applications of manual therapy.

\section{METHODS AND ANALYSIS}

\section{Source of data}

A prospective observational study will recruit a cohort of patients referred to the Italian Stomatologic Institute with a TMD diagnosis according to the Axis I of the Diagnostic Criteria for TMDs (DC/TMD). ${ }^{16}$ This protocol is written according to the Transparent Reporting of a multivariable prediction model for Individual Prognosis or Diagnosis statement ${ }^{17}$ in which recommendations are provided about prediction model development and validation. Ethical clearance will be obtained from the Ethics Committee of the Fondazione IRCCS Ca' Granda Ospedale Maggiore Policlinico, and the University of Birmingham Ethics Committee, and the study will be conducted in accordance with the Declaration of Helsinki.

Patient reported and physical assessment data will be collected at baseline prior to commencing treatment. Outcome will be collected at the end of the fourth session of craniomandibular manual therapy (at 1 month). This timeline has been selected based on previous studies investigating (1) the effects of manual therapy on pain ${ }^{18}{ }^{19}$; and (2) work confirming the effectiveness of manual therapy for TMD patients ${ }^{20}$ and is believed to be reasonable for the purposes of this study.

\section{Setting and participants}

Participant recruitment will be carried out at the TMJ Unit of the Italian Stomatological Institute (Dental Hospital) in Milan, Italy over a period of up to 12 months (planned start date July 2019). Consecutive eligible participants will be approached for recruitment until the sample size is reached.

\section{Eligibility criteria}

Inclusion criteria: (1) adults aged $\geq 18$ years; (2) TMD diagnosis according to the Diagnostic Criteria for TMDs $(\mathrm{DC} / \mathrm{TMD})^{16}$; (3) no therapeutic interventions reported (for their TMD) in the last 6 months ${ }^{21}$; (4) capacity to use and understand written and verbal Italian language; (5) mental capacity to provide informed consent.

Exclusion criteria: (1) TMD pain related to rheumatoid/inflammatory arthritis (2) any physical (eg, facial paralysis, neurological disorders, neuropathic pain) or mental condition (eg, cognitive deficit, mental illness and/or disorders) that could potentially influence the study results. Additionally, patients will be excluded if (3) they commence another treatment for their TMD 
(pharmacology, oral appliance, others) throughout the duration of the study.

\section{Recruitment}

Based on feasibility data from the last 5 years of activity at the TMJ Unit of Italian Stomatologic Institute, it is estimated that at least 130 eligible participants will be available for recruitment over 13 months. According to previous observational studies on the prediction of outcomes in musculoskeletal disorders, ${ }^{12}{ }^{13}$ it is estimated that $75 \%$ of eligible participants will consent to participation (100 participants).

All patients attending the TMJ Unit will be screened for the presence of a TMD. One expert dentist with $>10$ years' experience in the management of patients with TMD, will confirm the TMD diagnosis according to the DC/TMD using the Italian translation of the protocol. ${ }^{22}$ Subsequently, in accordance with the inclusion/exclusion criteria, he will explain the study to the potential participant and provide the patient information sheet. Participants will then give their written informed consent prior to inclusion in the study. Afterwards, the participant will be referred to see a physiotherapist (independent assessor, $>5$ years' experience in managing patients with TMD) for the baseline assessments (summarised in table 1) and then treatment will commence within the same week. After the last session (ie, 1 month from baseline), the participants will be assessed again by the assessing physiotherapist to measure outcome. Participant flow through the study is outlined in figure 1 .

\section{Treatment}

Participants will receive four sessions of manual therapy applied to craniomandibular structures over 4 weeks ${ }^{23-25}$. Two physiotherapists, each with $>5$ years' experience in manual therapy/TMD will perform the treatments. They will not be involved in participant recruitment, assessment or the collection of the outcome measure. Manual therapy techniques will be based on the clinical examination, and will be selected at the discretion of the treating physiotherapist according to their clinical reasoning of the individual case. Overall, the application of manual therapy aims to decrease pain by treating masticatory muscle trigger points, muscle tightness and restricted temporomandibular joint movements. Several techniques will be considered including: (1) ventral and caudal anterior glide temporomandibular joint mobilization $^{26}$; (2) soft tissue interventions for the management of trigger points in masticatory muscles ${ }^{27}$; (3) myofascial induction therapy (functional restoration of the fascial system) applied to craniomandibular structures. ${ }^{28}$

The structures targeted in the treatment sessions will be the temporomandibular joint, temporal muscles, masseter muscles, medial and lateral pterygoid muscles and suprahyoid muscles, applied at the discretion of the physiotherapist based on the patient's individual presentation. During the treatment sessions, the treating physiotherapists will provide explanations about the patient's condition and answer any participant questions by promoting general advice. The treatment sessions will last from 20 to $30 \mathrm{~min}$ duration. No other treatment (eg, oral appliance) will be performed for the management of their TMD. If during the course of the 4-week intervention, a patient seeks treatment for an acute episode of pain at another site (eg, neck pain, low back pain, shoulder pain) they will be withdrawn from the study.

\section{Outcome}

The outcome being predicted by the prediction model is pain intensity since patients with TMD typically report pain to be their primary problem, ${ }^{5}$ manual therapy is largely known to be effective principally for pain modulation ${ }^{29}$ and change in pain intensity has most commonly been the primary outcome of choice in several other studies of patients with TMD. ${ }^{30-33}$

Pain intensity will be calculated by averaging the ratings of current pain, average pain in the last week, and worst pain in the last week using the Visual Analogue Scale (VAS), consisting of a horizontal line measuring $10 \mathrm{~cm}$ (without marks), with 'no pain' written at the left extremity, and 'worst pain imaginable' at the right extremity. ${ }^{34}$ The VAS is a reliable and valid scale to assess pain intensity as an outcome measure in intervention studies. ${ }^{35}$ Based on the Initiative on Methods, Measurement, and Pain Assessment in Clinical Trials recommendations about TMD reviewed by Haythornthwaite, ${ }^{36}$ a reduction of at least $30 \%$ of the VAS score for pain intensity is considered clinically significant. Consequently, a reduction in the total VAS score ( $\geq 30 \%)$ will be defined as a good outcome. The outcome measure will be evaluated by the same independent assessor to minimise detection bias. ${ }^{37}$

To capture a potential change in function which may occur with a change in pain intensity, patients will also complete the patient-specific functional scale (PSFS) ${ }^{38}$ pretreatment and post-treatment. The PSFS is a selfreported outcome measure assessing functional change in patients with musculoskeletal disorders. ${ }^{39}{ }^{40} \mathrm{It}$ is responsive to clinically significant change over time. ${ }^{41}$ Patients will be invited to rate, on an 11-point scale, their level of difficulty performing at least three different daily activities. Following the treatment, patients will be required to score again the activities previously rated. The PSFS is a valid, reliable, and responsive outcome measure with a high test-retest reliability in different musculoskeletal disorders such as low back and neck pain. ${ }^{42-44}$

\section{Candidate predictors}

The candidate predictors that have been chosen are reliable and valid measures which have a relationship with pain. The selection is based on previous research on prognostic factors for TMD and altered pain modulation in musculoskeletal disorders. ${ }^{45}{ }^{46}$ Candidate predictors are summarised in table 1, with further detail in online supplementary file S1. All data collection will be standardised through protocols and clinical report forms. 
Table 1 Summary of candidate predictors

\begin{tabular}{ll}
\hline Domain / candidate predictor & Measure / data item \\
\hline $\begin{array}{l}\text { Demographical variables } \\
\text { Age }\end{array}$ & Years \\
$\begin{array}{l}\text { Gender } \\
\text { Education }\end{array}$ & Female / male \\
$\begin{array}{l}\text { General health variables } \\
\text { Health-related quality of life }\end{array}$ & $\begin{array}{l}\text { EuroQol EQ-5D-5L }{ }^{52} \\
\text { Sleep quality }\end{array}$ \\
$\begin{array}{l}11-\text { point }(0-10) \text { Numerical Rating Scales, relating to current pain, from 'best possible } \\
\text { sleep' to 'worst possible sleep'53 }\end{array}$
\end{tabular}

Psychosocial features

Coping strategies applied during Coping Strategies Questionnaire 27 (CSQ-27) $)^{54}$

a painful experience

Anxiety and depression

Hospital Anxiety and Depression Scales (HADS) ${ }^{55}$

Treatment expectation Positive / negative expectation ${ }^{56}$

TMD characteristics

\begin{tabular}{ll} 
Pain duration & Days \\
Pain intensity & VAS: averaging ratings of current pain, average pain and worst pain in the last week \\
\hline Pain location & Pain drawing as described in the protocol of Diagnostic Criteria for TMD (DC/TMD) \\
\hline Central sensitisation & Central Sensitisation Inventory (CS) ${ }^{58}$ \\
Classification of TMD & In according to DC/TMD Taxonomy \\
Oral behaviours & Oral Behaviours Checklist (OBC) \\
$\begin{array}{l}\text { Characteristic pain intensity and } \\
\text { disability }\end{array}$ & $\begin{array}{l}\text { Graded Chronic Pain Scale (GCPS) version 2.0 (Italian version-www.rdc- } \\
\text { tmdinternational.org) }\end{array}$
\end{tabular}

Temporomandibular joint (TMJ) and masticatory muscles clinical test

TMJ range of motion Maximal Mouth Opening (MMO) without pain measured in $\mathrm{mm}$ through a ruler as described in the DC/TMD protocol ${ }^{16}$

TMJ palpation pain Dynamic TMJ lateral pole palpation ( $1 \mathrm{~kg}$ of palpation pressure) in according to DC/TMD protocol $^{16}$

Score range: $0-1$ (no pain=0; pain=1)

Muscle palpation pain Palpation in the following six bilateral points: lateral pterygoid area $(0.5 \mathrm{~kg}$ intraoral palpation), temporalis tendon $(0.5 \mathrm{~kg}$ intraoral palpation), masseter muscle $(1 \mathrm{~kg}$ extraoral palpation) as described in the DC/TMD protocol. ${ }^{16}$ Score range: $0-1$ ( $<3$ sites with familiar pain $=0 ; \geq 3$ sites with familiar pain $=1$ )

JAw-test Immediate effects of brief intraoral MT techniques on pain (VRS) and TMJ range of motion (MMO). A standardised procedure is fully described in online supplementary file S1.

Score range $0-2:(0=$ no change; $1=$ pain improvement or $\mathrm{MMO}$ improvement; $2=$ improvement of both)

VAS, Visual Analogue Scale; VRS, Verbal Rating Scale.

\section{Data handling}

Candidate predictors will be collected by independent physiotherapist assessor. All data will be confidentially secured by storing it on a password-protected computer attainable only by the principal investigators (GA). All individual details will be replaced with ID codes. At the end of the data collection, all data stored on the principal investigator's computer will be transferred securely to a server at the Centre of Precision Rehabilitation for Spinal Pain at Birmingham University where the data will be analysed. All data will be stored on a secure server at the University of Birmingham for a period of 10 years in line with Research Governance procedures. Data will be analysed using IBM SPSS Statistics V.25.

\section{Sample size}

Exploratory factor analysis will be utilised to reduce the number of predictors. ${ }^{47}$ This method will guarantee an adequate sample size (at least 10 cases per candidate predictor) to power the final regression analysis. ${ }^{48}{ }^{49}$ Data will be collected for a sample size of 100 participants so 


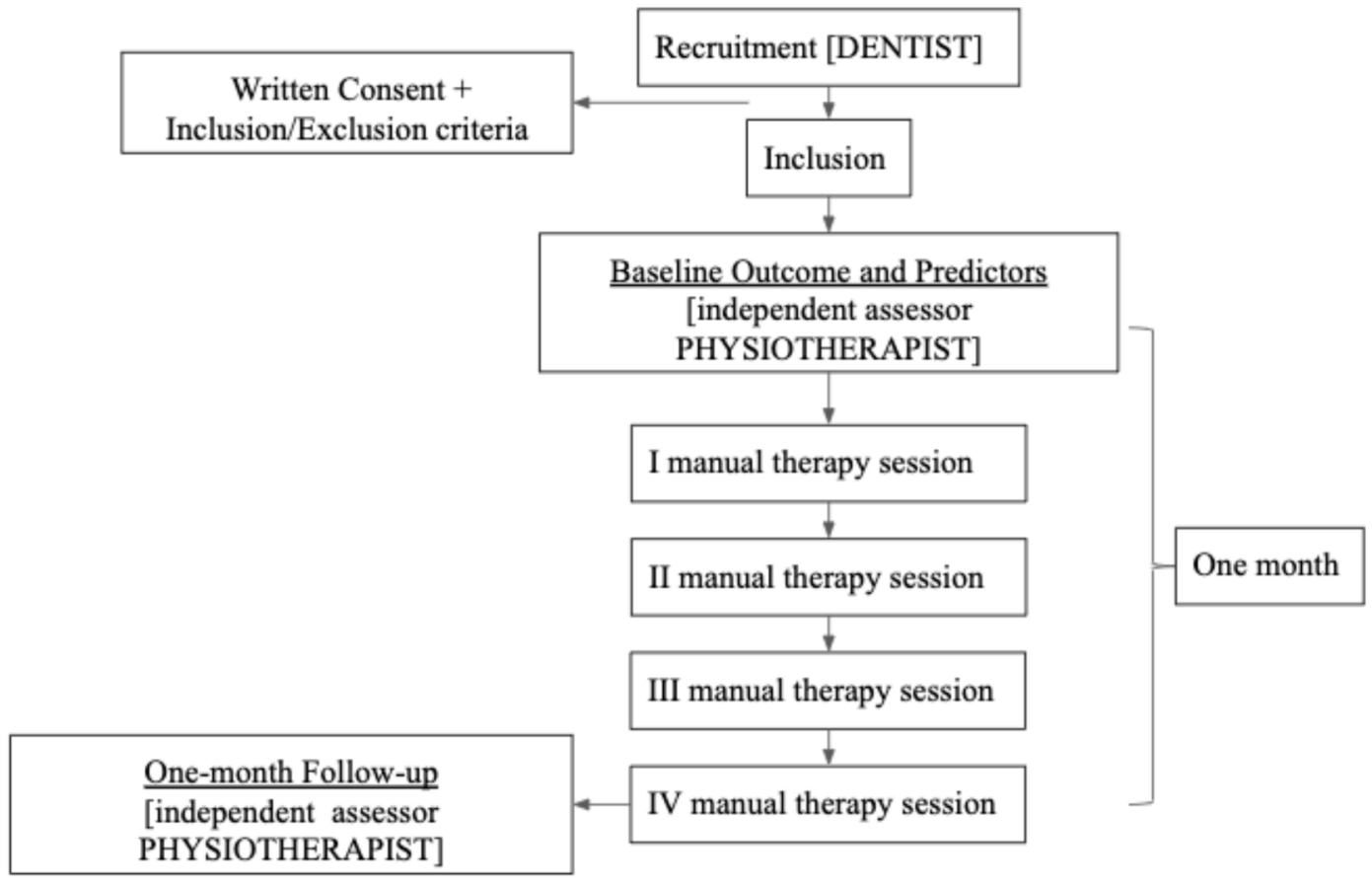

Figure 1 Participant flow through the study.

that, considering $10 \%$ of potential drops out, final data are available for 90 participants.

\section{Statistical analysis methods}

A flow diagram will report eligible participants, examined for eligibility, confirmed eligible, recruited into the study, completed follow-up and analysed. Reasons for nonparticipation, exclusion, drop-outs and withdrawal will be fully documented and all missing data of participants will be reported. Participant characteristics (candidate predictors-table 1) will be summarised with a descriptive method.

A primary phase of the exploratory data analysis will summarise data to implement the predictive model. ${ }^{50}$ Multicollinearity between candidate predictors will be assessed at baseline. Outcome (VAS pain intensity) will be split into good versus poor as described previously (good outcome: reduction in VAS score $\geq 30 \%)$. ${ }^{36}$ Exploratory factor analysis will be applied to analyse factor loading of candidate predictors (summary scores) on good outcome at 1 month. This process will reduce candidate predictors (supported by the cohort sample of 90) to enter into the final model.

The statistical model has been designed a priori. To investigate the impact of each predictive factor on good outcome, a logistic multivariable regression model will be performed. For each candidate predictor, the mean differences or the OR with their 95\% CIs will be calculated. A multiple imputation analysis ${ }^{51}$ will be applied to manage possible missing data. The multivariable analysis will initially consider all candidate predictors. In the case of a high correlation between candidate predictors, a reduced multivariate analysis will be considered.

\section{DISCUSSION}

There is a need to identify predictors for pain reduction in patients with TMD following specific treatments in order to inform clinical decision-making. Several therapies are described for patients with TMD such as the use of oral appliances, different types of physical therapy modalities, pharmacology or temporomandibular joint arthrocentesis yet the amount of pain relief that different people receive from each intervention is variable. ${ }^{710}$ As shown by Forssell et $a l^{14}$ and Kapos et al, ${ }^{15}$ many patients continue to experience pain following such interventions. Investigating factors associated with pain relief to such treatments can facilitate clinical assessment and treatment selection.

Physical therapy is one of the most common conservative interventions to treat $\mathrm{TMD}^{7}$ Among different physical therapy modalities, manual therapy can provide symptom and functional improvements ${ }^{10}$ including pain relief. ${ }^{1131}$ Knowledge of predictive factors associated with good outcome to a specific intervention such as manual therapy applied to craniomandibular structures will facilitate clinical decision-making. Ultimately, such knowledge will lead to improved clinical and cost effectiveness of rehabilitation approaches.

\section{Quality assurance}

Only participants that have not received therapeutic intervention for their TMD in the last six months will be included in the current study. It is possible that such eligibility criteria could generate selection bias. To address this potential bias, the number of eligible and included subjects with the reason for non-participation will be documented. 


\section{Patient and public involvement}

The research question in this study was developed following consultations and discussion with patients. Patients will not be involved in the analysis and data collection but will contribute to data interpretation and production of a lay summary of the findings.

\section{Ethics and dissemination}

The research protocol has been submitted to the Ethics Committee of the 'Fondazione IRCCS Ca' Granda Ospedale Maggiore Policlinico' and subsequently will be submitted to the University of Birmingham Ethics Committee for approval. Researchers will inform all participants on the characteristics of the research and will obtain written consent. Participants will be informed that they are free to withdraw from the study at any time, without needing to provide reason. Any concerns for a participant by the study team will be fed back to the primary investigator (GA). Baseline characteristics of withdrawn participants will be compared with those of retained participants to assess for any differences. In the event of any unlikely adverse events, this will be immediately reported by the principal investigator to the ethics committee.

The results of this study will submitted for publication in a peer review journal and presented at conferences.

\section{Limitations}

The study could potentially generate a non-representative sample of patients with TMD due to a possible selection bias. Subjects reporting other treatments before $(6$ months) and during the study will be excluded to minimise confounding bias and preserve internal validity. This could potentially generate a non representative sample of TMDs because of exclusion of patients with high levels of pain which seek additional treatment. This potential event, associated with the fact that this observational study will be performed at a single site only, could reduce the external validity and the generalisability of the results.

\section{CONCLUSION}

This protocol paper describes what will be the first study to identify factors associated with pain reduction following manual therapy in patients with TMD. It is anticipated that the knowledge gained from the study described within this protocol, will facilitate clinical decision-making for manual therapists managing patients with TMD.

Twitter Giacomo Asquini @AsquiniGiacomo, Nicola R Heneghan @HeneghanNicola, Alison B Rushton @abrushton and Deborah Falla @Deb_Falla

Contributors GA, AEB and DF formulated the research question and study focus. GA drafted the initial version of the manuscript with DF. NRH, ABR, GB and ML provided guidance on topic, methodology and analyses. All authors reviewed and commented on each draft of the protocol. All authors have approved the final manuscript. DF is guarantor.

Funding The authors have not declared a specific grant for this research from any funding agency in the public, commercial or not-for-profit sectors.

Competing interests None declared.
Patient consent for publication Not required.

Provenance and peer review Not commissioned; externally peer reviewed.

Open access This is an open access article distributed in accordance with the Creative Commons Attribution Non Commercial (CC BY-NC 4.0) license, which permits others to distribute, remix, adapt, build upon this work non-commercially, and license their derivative works on different terms, provided the original work is properly cited, appropriate credit is given, any changes made indicated, and the use is non-commercial. See: http://creativecommons.org/licenses/by-nc/4.0/.

\section{ORCID iDs}

Nicola R Heneghan http://orcid.org/0000-0001-7599-3674

Alison B Rushton http://orcid.org/0000-0001-8114-7669

Deborah Falla http://orcid.org/0000-0003-1689-6190

\section{REFERENCES}

1 Lipton JA, Ship JA, Larach-Robinson D. Estimated prevalence and distribution of reported orofacial pain in the United States. J Am Dent Assoc 1993;124:115-21.

2 NIDCR, National Institute of Health. Facial pain (2014). Available: http://www.nidcr.nih.gov/DataStatistics/FindDataByTopic/FacialPain/ [Accessed 28 Dec 2018].

3 Montero J, Llodra J-C, Bravo M. Prevalence of the signs and symptoms of temporomandibular disorders among Spanish adults and seniors according to five national surveys performed between 1993 and 2015. J Oral Facial Pain Headache 2018;32:349-57.

4 Adèrn B, Minston A, Nohlert E, et al. Self-reportance of temporomandibular disorders in adult patients attending general dental practice in Sweden from 2011 to 2013. Acta Odontol Scand 2018;76:530-4

5 De Leeuw R, Klasser GD. Orofacial pain guidelines for assessment, diagnosis, and management. 5th ed. Hanover park, II: Quintessence publishing, 2013.

6 Plesh O, Adams SH, Gansky SA. Temporomandibular joint and muscle disorder-type pain and comorbid pains in a national US sample. J Orofac Pain 2011;25:190-8.

7 Calixtre LB, Grüninger BLdaS, Haik MN, et al. Effects of cervical mobilization and exercise on pain, movement and function in subjects with temporomandibular disorders: a single group pre-post test. J Appl Oral Sci 2016;24:188-97.

8 Slade GD, Bair E, Greenspan JD, et al. Signs and symptoms of firstonset TMD and sociodemographic predictors of its development: the OPPERA prospective cohort study. J Pain 2013;14:T20-32.

9 Coskun Benlidayi I, Salimov F, Kurkcu M, et al. Kinesio taping for temporomandibular disorders: single-blind, randomized, controlled trial of effectiveness. J Back Musculoskelet Rehabil 2016;29:373-80.

10 Armijo-Olivo S, Pitance L, Singh V, et al. Effectiveness of manual therapy and therapeutic exercise for temporomandibular disorders: systematic review and meta-analysis. Phys Ther 2016;96:9-25.

11 Kalamir A, Graham PL, Vitiello AL, et al. Intra-Oral myofascial therapy versus education and self-care in the treatment of chronic, myogenous temporomandibular disorder: a randomised, clinical trial. Chiropr Man Therap 2013;21.

12 Cleland JA, Childs JD, Fritz JM, et al. Development of a clinical prediction rule for guiding treatment of a subgroup of patients with neck pain: use of thoracic spine manipulation, exercise, and patient education. Phys Ther 2007;87:9-23.

13 Flynn T, Fritz J, Whitman J, et al. A clinical prediction rule for classifying patients with low back pain who demonstrate short-term improvement with spinal manipulation. Spine 2002;27:2835-43.

14 Forssell H, Kauko T, Kotiranta U, et al. Predictors for future clinically significant pain in patients with temporomandibular disorder: a prospective cohort study. Eur J Pain 2017;21:188-97.

15 Kapos FP, Look JO, Zhang L, et al. Predictors of long-term temporomandibular disorder pain intensity: an 8-year cohort study. $J$ Oral Facial Pain Headache 2018;32:113-22.

16 Schiffman E, Ohrbach R, Truelove E, et al. Diagnostic criteria for temporomandibular disorders (DC/TMD) for clinical and research applications: recommendations of the International RDC/TMD Consortium network and orofacial pain special interest group. $J$ Oral Facial Pain Headache 2014;28:6-27.

17 Collins GS, Reitsma JB, Altman DG, et al. Transparent reporting of a multivariable prediction model for individual prognosis or diagnosis (TRIPOD): the TRIPOD statement'. BMC Medicine 2015;350:g7594.

18 Bishop MD, Torres-Cueco R, Gay CW, et al. What effect can manual therapy have on a patient's pain experience? Pain Manag 2015;5:455-64. 
19 Vigotsky AD, Bruhns RP. Corrigendum to "The role of descending modulation in manual therapy and its analgesic implications: a narrative review". Pain Res Treat 2017;2017.

20 Calixtre LB, Moreira RFC, Franchini GH, et al. Manual therapy for the management of pain and limited range of motion in subjects with signs and symptoms of temporomandibular disorder: a systematic review of randomised controlled trials. J Oral Rehabil 2015;42:847-61.

21 Wahlund K, Nilsson I-M, Larsson B. Treating temporomandibular disorders in adolescents: a randomized, controlled, sequential comparison of relaxation training and occlusal appliance therapy. $J$ Oral Facial Pain Headache 2015;29:41-50.

22 Ohrbach R, Michelotti A, Segù M, et al. Diagnostic Criteria for Temporomandibular Disorders: Assessment Instruments. Version 15May2016. [Criteri diagnostici per i disordini temporomandibolari: Strumenti valutativi (DC/TMD), 2017. Available: www.rdctmdinternational.org [Accessed 31 Mar 2019].

23 Crockett DJ, Foreman ME, Alden L, et al. A comparison of treatment modes in the management of myofascial pain dysfunction syndrome. Biofeedback Self Regul 1986;11:279-91.

24 Guarda-Nardini L, Stecco A, Stecco C, et al. Myofascial pain of the jaw muscles: comparison of short-term effectiveness of botulinum toxin injections and fascial manipulation technique. Cranio 2012;30:95-102.

25 Nascimento M-M, Vasconcelos B-C, Porto G-G, et al. Physical therapy and anesthetic blockage for treating temporomandibular disorders: a clinical trial. Med Oral Patol Oral Cir Bucal 2013;18:e81-5.

26 Cleland J, Palmer J. Effectiveness of manual physical therapy, therapeutic exercise, and patient education on bilateral disc displacement without reduction- of the temporomandibular joint: a single-case design. J Orthop Sports Phys Ther 2004;34:535-48.

27 Miernik M, Wieckiewicz M, Paradowska A, et al. Massage therapy in myofascial TMD pain management. Adv Clin Exp Med 2012;21:681-5.

28 Fernandez-de-las-Pena C, Mesa-Jimenez J. Temporomandibular disorders: manual therapy, exercise and needling. United Kingdom: Handspring Publishing, 2018.

29 Bialosky JE, Bishop MD, Price DD, et al. The mechanisms of manual therapy in the treatment of musculoskeletal pain: a comprehensive model. Man Ther 2009;14:531-8.

30 Kalamir A, Pollard H, Vitiello A, et al. Intra-Oral myofascial therapy for chronic myogenous temporomandibular disorders: a randomized, controlled pilot study. J Man Manip Ther 2010;18:139-46.

31 Gomes CAFdeP, Politti F, Andrade DV, et al. Effects of massage therapy and occlusal splint therapy on mandibular range of motion in individuals with temporomandibular disorder: a randomized clinical trial. J Manipulative Physiol Ther 2014;37:164-9.

32 Tuncer AB, Ergun N, Tuncer AH, et al. Effectiveness of manual therapy and home physical therapy in patients with temporomandibular disorders: a randomized controlled trial. J Bodyw Mov Ther 2013;17:302-8.

33 von Piekartz H, Hall T. Orofacial manual therapy improves cervical movement impairment associated with headache and features of temporomandibular dysfunction: a randomized controlled trial. Man Ther 2013;18:345-50.

34 Haefeli M, Elfering A. Pain assessment. Eur Spine J 2006;15 Suppl 1:S17-24.

35 Dworkin RH, Turk DC, Farrar JT, et al. Core outcome measures for chronic pain clinical trials: IMMPACT recommendations. Pain 2005;113:9-19.

36 Haythornthwaite JA. IMMPACT recommendations for clinical trials: opportunities for the RDC/TMD. J Oral Rehabil 2010;37:799-806.

37 Higgins JPT, Altman DG, Gøtzsche PC, et al. The Cochrane collaboration's tool for assessing risk of bias in randomised trials. BMJ 2011;343.

38 Stratford Pet al. Assessing disability and change on individual patients: a report of a patient specific measure. Physiotherapy Canada 1995;47:258-63.
39 Horn KK, Jennings S, Richardson G, et al. The patient-specific functional scale: psychometrics, clinimetrics, and application as a clinical outcome measure. J Orthop Sports Phys Ther 2012;42:30-D17.

40 Abbott JH, Schmitt J. Minimum important differences for the patientspecific functional scale, 4 region-specific outcome measures, and the numeric pain rating scale. J Orthop Sports Phys Ther 2014;44:560-4.

41 Maughan EF, Lewis JS. Outcome measures in chronic low back pain Eur Spine J 2010:19:1484-94.

42 Hefford C, Abbott JH, Arnold R, et al. The patient-specific functional scale: validity, reliability, and responsiveness in patients with upper extremity musculoskeletal problems. J Orthop Sports Phys Ther 2012;42:56-65.

43 Westaway MD, Stratford PW, Binkley JM. The patient-specific functional scale: validation of its use in persons with neck dysfunction. J Orthop Sports Phys Ther 1998;27:331-8.

44 Chatman AB, Hyams SP, Neel JM, et al. The patient-specific functional scale: measurement properties in patients with knee dysfunction. Phys Ther 1997;77:820-9.

45 Bair E, Gaynor S, Slade GD, et al. Identification of clusters of individuals relevant to temporomandibular disorders and other chronic pain conditions: the OPPERA study. Pain 2016;157:1266-78.

46 Clark J, Nijs J, Yeowell G, et al. What are the predictors of altered central pain modulation in chronic musculoskeletal pain populations? A systematic review. Pain Physician 2017;20:487-500.

47 Fabrigar LR, Wegener DT, MacCallum RC, et al. Evaluating the use of exploratory factor analysis in psychological research. Psychol Methods;4:272-99.

48 Peduzzi P, Concato J, Kemper E, et al. A simulation study of the number of events per variable in logistic regression analysis. $J$ Clin Epidemiol 1996;49:1373-9.

49 Vittinghoff E, McCulloch CE. Relaxing the rule of ten events per variable in logistic and COX regression. Am J Epidemiol 2007:165:710-8.

50 Shmueli G. To explain or to predict? Statistical Science 2010;25:289-310.

51 Sterne JAC, White IR, Carlin JB, et al. Multiple imputation for missing data in epidemiological and clinical research: potential and pitfalls. BMJ 2009;338.

52 Brooks R. EuroQol: the current state of play. Health Policy 1996;37:53-72.

53 Cappelleri JC, Bushmakin AG, McDermott AM, et al. Psychometric properties of a single-item scale to assess sleep quality among individuals with fibromyalgia. Health Qual Life Outcomes 2009;7:54.

54 Monticone M, Ferrante S, Giorgi I, et al. The 27-item coping strategies questionnaire-revised: confirmatory factor analysis, reliability and validity in Italian-speaking subjects with chronic pain. Pain Res Manag 2014;19:153-8.

55 Zigmond AS, Snaith RP. The hospital anxiety and depression scale. Acta Psychiatr Scand 1983;67:361-70.

56 Puentedura EJ, Cleland JA, Landers MR, et al. Development of a clinical prediction rule to identify patients with neck pain likely to benefit from thrust joint manipulation to the cervical spine. J Orthop Sports Phys Ther 2012;42:577-92.

57 Davis CE, Stockstill JW, Stanley WD, et al. Pain-Related worry in patients with chronic orofacial pain. J Am Dent Assoc 2014;145:722-30.

58 Mayer TG, Neblett R, Cohen $\mathrm{H}$, et al. The development and psychometric validation of the central sensitization inventory. Pain Pract 2012;12:276-85.

59 Peck CC, Goulet J-P, Lobbezoo F, et al. Expanding the taxonomy of the diagnostic criteria for temporomandibular disorders. $J$ Oral Rehabil 2014;41:2-23.

60 Ohrbach R, Markiewicz MR, McCall WD. Waking-state oral parafunctional behaviors: specificity and validity as assessed by electromyography. Eur J Oral Sci 2008;116:438-44. 